\title{
Evolución del neurodesarrollo de gemelos dicigóticos con síndrome de Waardenburg e hipoacusia neurosensorial
}

\author{
Emilio Arch-Tirado, ${ }^{*}$ Lucía Arellano-Cabrera, ${ }^{\ddagger}$ Ileana Gutiérrez-Farfán, ${ }^{\ddagger}$ S Alejandra Aguilera-Tello, ${ }^{\ddagger}$ \\ Miguel Ángel Collado-Corona,* Ana Luisa Lino-González ${ }^{\S}$ \\ * Centro Neurológico, Centro Médico ABC Santa Fe. Ciudad de México, México. \\ ¥ Servicio de Audiología. Postgrado de Alta Especialidad de Audiología Pediátrica. Instituto Nacional \\ de Rehabilitación "Luis Guillermo Ibarra Ibarra”. Ciudad de México, México. \\ § Subdirección de Investigación Clínica, Servicio de Neurociencia Clínica, Área de Discapacidad del Lenguaje, Voz y \\ Audición. Instituto Nacional de Rehabilitación “Luis Guillermo Ibarra Ibarra”. Ciudad de México, México.
}

\section{RESUMEN}

\begin{abstract}
Antecedentes. El síndrome de Waardenburg es una enfermedad genética poco común; uno de sus hallazgos clínicos más significativos para determinarla es la hipoacusia neurosensorial. Objetivo: Describir la evolución del neurodesarrollo de gemelos dicigóticos con síndrome de Waardenburg e hipoacusia neurosensorial. Métodos: Estudio descriptivo en el que participaron gemelos dicigóticos de cuatro años y seis meses con síndrome de Waardenburg e hipoacusia neurosensorial bilateral. Se recopiló aquella información relacionada con antecedentes familiares, perinatales, evaluaciones previas, tratamiento y evolución de los pacientes. Para los resultados cuantitativos obtenidos a través de la Guía Portage de Educación Preescolar, se construyó una tabla y se calculó $\chi^{2}$ con un nivel de confianza de $p<0.05$. Resultados: El gemelo de sexo femenino mostró una mejor evolución en su neurodesarrollo. Conclusiones: Estudiar a poblaciones de gemelos aporta información valiosa que favorece un mayor control de las variables ambientales, educativas y atencionales.
\end{abstract}

Palabras clave: Síndrome Waardenburg, gemelos dicigóticos, hipoacusia neurosensorial, neurodesarrollo.

\begin{abstract}
Background: Waardenburg syndrome is a rare genetic disease and one of its most significant clinical findings is neurosensory hearing loss. Objective: To describe the neurodevelopment evolution of dizygotic twins with Waardenburg syndrome and sensorineural hearing loss. Methods: Descriptive study, involved dizygotic twins of 4 years 6 months, with Waardenburg syndrome and bilateral sensorineural hearing loss. Information was collected related to family, perinatal, previous evaluations, treatment and evolution of patients. For the quantitative results obtained in the Portage Guide for preschool education, a table was constructed and $\chi^{2}$ was calculated with a confidence level for $p<0.05$. Results: The female twin showed better evolution in her neurodevelopment. Conclusions: Studying populations of twins provides valuable information by promoting greater control of environmental, educational and attentional variables.
\end{abstract}

Keywords: Waardenburg syndrome, dizygotic twins, sensorineural hearing loss, neurodevelopment.

\section{INTRODUCCIÓN}

\author{
Abreviaturas: \\ CC = Cuerpo calloso. \\ EOAsT $=$ Emisiones otoacústicas transientes. \\ EDP-BL = Escala de desarrollo psicomotor Brunet-Lézine. \\ FIESTA = Fast imaging employing steady state. \\ $\mathrm{GF}=$ Gemelo femenino. \\ $\mathrm{GM}=$ Gemelo masculino. \\ GPEP = Guía Portage de educación preescolar. \\ HNS = Hipoacusia neurosensorial. \\ IC = Implante coclear. \\ INRLGII = Instituto Nacional de Rehabilitación "Luis Guillermo \\ Ibarra Ibarra". \\ PEAEE $=$ Potenciales evocados auditivos de estado estable. \\ PEATC $=$ Potenciales evocados auditivos de tallo cerebral. \\ $\mathrm{RM}=$ Resonancia magnética. \\ $\mathrm{SNC}=$ Sistema nervioso central. \\ SW = Síndrome de Waardenburg.
}

El síndrome de Waardenburg (SW) es una enfermedad genética que se caracteriza por diversos grados de hipoacusia neurosensorial (HNS), pigmentación anor$\mathrm{mal}^{1}$ y defectos estructurales derivados de las células de la cresta neural. ${ }^{2,3}$ Este síndrome se considera como un trastorno auditivo-pigmentario originado por una deficiencia en la diferenciación de melanocitos y la migración de la cresta neural embrionaria, la cual afecta piel, pelo, ojos, estría vascular de la cóclea y órgano de Corti. Los melanocitos se encargan de mantener la composición iónica de la endolinfa coclear en la estría vascular, y su ausencia provoca un deterioro de la función endococlear, lo que da como resultado un colapso de la membrana de Reissner y la destrucción 
de las células ciliadas, con la consecuente generación de HNS. ${ }^{2,4}$

El SW es un trastorno genético poco común ${ }^{2-4}$ con rasgo autosómico dominante ${ }^{4-6}$ y de penetrancia y expresividad variable. ${ }^{2,5} \mathrm{Su}$ incidencia reportada es de 1/42,000 sujetos, ${ }^{2,7}$ y afecta por igual a ambos sexos y a todas las razas. ${ }^{1,2}$ Éste se clasifica en cuatro tipos $^{1,6,7}$ y se suele relacionar con los genes PAX3, MITF, SOX10, EDN3, EDNRB y SNAI2. ${ }^{1-3,6,8}$ El diagnóstico es clínico mediante la detección de uno o dos criterios mayores y dos menores; a saber, ${ }^{1,5}$ se consideran criterios mayores: HNS congénita, anormalidades en la pigmentación del iris (completa o segmentada), poliosis, desplazamiento lateral del canto interno ocular y familiar de primer grado con SW. Para los criterios menores, se consideran: puente nasal ancho, sinofridia, canicie prematura, hipoplasia de las alas nasales y leucodermia congénita. ${ }^{4}$

La HNS es el hallazgo clínico más significativo. Esta pérdida auditiva está presente en $60 \%$ de los pacientes con SW tipo 1 y en $90 \%$ del tipo $2,{ }^{6,7}$ por lo general es bilateral y no necesariamente simétrica. ${ }^{6,7,9}$ De la misma manera, los problemas visuales también son frecuentes en estos pacientes; este aspecto, hace necesario realizar exámenes visuales detallados. Los signos oculares que más se han reportado son glaucoma de ángulo abierto, oclusión de la vena retiniana y ambliopía. ${ }^{4}$ En la literatura médica, se reporta una prevalencia de $45.8 \%$ de patologías oftalmológicas en niños hipoacúsicos. ${ }^{10}$ Asimismo, se ha sugerido que estas elevadas tasas se atribuyen a que la maduración de la retina y la cóclea se llevan a cabo durante la cuarta semana de gestación a partir de la misma capa embrionaria (ectodermo), pudiendo ser susceptibles a factores genéticos $y / 0$ ambientales. ${ }^{11} \mathrm{Si}$ esta doble discapacidad no es identificada tempranamente, el desarrollo de habilidades lingüísticas y comunicativas se verá limitado, debido a que los hipoacúsicos requieren del sentido de la vista para desarrollar habilidades de comunicación eficientes. ${ }^{10-12}$

El dimorfismo sexual de la anatomía cerebral se produce por la influencia de acciones hormonales reguladas por la expresión genética. Esta expresión emana de la estructuración del sexo durante los primeros cuatro meses de gestación (sin olvidar otros factores determinantes, como el entorno y la edad). Al observar diferencias cognitivas y conductuales entre ambos sexos, es posible inferir discrepancias anatómicas, bioquímicas, histológicas y fisiológicas a nivel de sistema nervioso central (SNC). En lo que respecta a la corteza cerebral, la densidad neuronal es más alta y de mayor diámetro craneal en los hombres, en tanto que en las mujeres se observa un incremento recíproco del neuropilo y de los procesos neuronales. Estas diferencias entre corteza derecha e izquierda (asimetría) son comunes en los hombres, pero no así en las mujeres. De la misma manera, estas diferencias explican contrastes en el desempeño de ambos sexos para algunas habilidades y capacidades cognitivas. Se sugiere que los hombres se desempeñan mejor en tareas visoespaciales, las cuales son ejecutadas principalmente por el hemisferio derecho y que las mujeres sobresalen en habilidades del lenguaje por la participación de ambos hemisferios en el procesamiento de la información lingüística y la generación de mayor arborización dendrítica.

Con respecto al cuerpo calloso (CC), se refiere que, por una parte, en los hombres es más voluminoso y que la parte posterior en las mujeres (esplenio) es más bulbosa y es significativamente más grande; por otra parte, se ha reportado que la adherencia intertalámica está presente en $68 \%$ de los hombres y en $78 \%$ de las mujeres. ${ }^{13}$ Estudios con resonancia magnética (RM) de alta resolución estructural han corroborado que el CC de las mujeres es más voluminoso en la porción posterior; además, estudios con RM de alta resolución con tensor de difusión han evidenciado una mayor densidad de fibras nerviosas en las mujeres y una disminución de la mielinización en los hombres. ${ }^{14}$

El objetivo del presente trabajo es describir la evolución del neurodesarrollo de gemelos dicigóticos con síndrome de Waardenburg e hipoacusia neurosensorial.

\section{Pacientes y MÉtodos}

Se trata de un estudio descriptivo en el que participaron un par de gemelos dicigóticos: uno del sexo femenino (GF) y otro del sexo masculino (GM), cuya

Tabla 1: Condiciones perinatales de los pacientes.

\begin{tabular}{lrr}
\hline & GF & GM \\
\hline $\begin{array}{lr}\text { Peso al nacer } \\
\text { (g) }\end{array}$ & 2,800 & \\
Talla (cm) & 50 & 3,200 \\
Apgar & $8 / 8$ & 49 \\
\hline
\end{tabular}

GF = gemelo femenino; GM = gemelo masculino . 
Tabla 2: Resultados de la escala de desarrollo Brunet-Lézine.

\begin{tabular}{lcc}
\hline & GF & GM \\
\hline Cociente de desarrollo & 80 & 70 \\
Edad de desarrollo & 1 año, 8 meses & 1 año, 5 meses \\
Retraso desarrollo & 5 meses & 8 meses \\
Nivel de retraso & Moderado & Grave \\
Postura & Normal & Deficiente \\
Coordinación & Deficiente & Deficiente \\
Lenguaje & Muy deficiente & Muy deficiente \\
Sociabilidad & Deficiente & Muy deficiente \\
\hline
\end{tabular}

$\mathrm{GF}$ = gemelo femenino; GM = gemelo masculino.

edad era al inicio del estudio de cuatro años y seis meses. Ambos habían sido diagnosticados con SW e HNS profunda bilateral. Los gemelos fueron producto de cuarta gesta, obtenidos a término vía cesárea, quienes presentaron llanto y respiración espontáneos y distintas condiciones perinatales (Tabla 1).

El protocolo fue aprobado por los Comités de Investigación y Ética del Instituto Nacional de Rehabilitación "Luis Guillermo Ibarra Ibarra" (INRLGII), además de que, acorde con la Declaración de Helsinki, se le pidió a la madre su firma del consentimiento informado.

Al realizar la revisión de los expedientes, se encontró que a la edad de un año y nueve meses los pacientes ingresaron al Área de Audiología del INRLGII. En dicha institución, se efectuó la historia clínica completa. Para obtener los umbrales auditivos, se realizó: a) audiometría por observación de la conducta en las frecuencias 500, 2,000 y $4,000 \mathrm{~Hz}$ a una intensidad máxima de $70 \mathrm{~dB}$; b) emisiones otoacústicas transientes (EOAsT) para determinar funcionalidad de las células ciliadas externas; c) timpanometría para determinar cambios de compliancia de la membrana timpánica y cadena osicular utilizando un tono a $226 \mathrm{~Hz}$, considerando como parámetro de normalidad la curva tipo A de Jerger; d) potenciales evocados auditivos de tallo cerebral (PEATC) para obtener la respuesta electrofisiológica de latencia corta generada por estructuras neurales del nervio auditivo, tallo cerebral y componentes talámicos mediante frecuencias agudas, y e) potenciales evocados auditivos de estado estable (PEAEE) buscando respuestas cuasi-sinusoidales en las frecuencias de 500, 1,000, 2,000 y 4,000 Hz.
Para determinar la condición estructural se realizó una tomografía computarizada de cráneo con atención a fosa posterior y RM, así como una evaluación oftalmológica para detectar si había problemas visuales. A la edad reportada al ingreso (1 año, 9 meses), el Área de Psicología realizó una evaluación con la escala de desarrollo psicomotor de Brunet-Lézine (EDP-BL), ${ }^{15}$ la cual se encarga de determinar el cociente de desarrollo del niño al comparar la edad cronológica real con la edad de desarrollo obtenido. Ésta abarca las áreas: a) postura, b) coordinación, c) lenguaje y d) sociabilidad, las cuales son aplicables desde el nacimiento hasta los seis años.

A la edad de dos años y 11 meses, se les realizó cirugía para colocar un implante coclear (IC) en su oído derecho y se inició terapia del lenguaje con el método auditivo verbal con frecuencia de dos sesiones semanales. Asimismo, se comenzó con un estricto monitoreo audiológico para determinar el funcionamiento del IC y asegurar una adecuada discriminación auditiva.

A los cuatro años y seis meses, los gemelos fueron valorados con la Guía Portage de Educación Preescolar (GPEP), ${ }^{16}$ la cual evalúa cinco áreas del desarrollo desde el nacimiento hasta los seis años a través de una lista de objetivos basados en patrones de crecimiento y desarrollo normal. Las áreas evaluadas fueron: lenguaje, cognición, área psicomotriz y social. La prueba se aplicó a cada gemelo por separado en presencia de la madre; a ellos se les dio instrucciones con apoyo de lenguaje oral, gestos y lenguaje corporal para asegurar la comprensión y la ejecución de las tareas indicada. En los casos en los que se observó dificultad por falta de entendimiento, se solicitó a la madre que informa- 
ra si anteriormente observó alguna de las conductas presentadas en ellos; al ser notorio un retraso en el desarrollo durante la aplicación del instrumento, se decidió evaluar el área del lenguaje a partir de los dos años y la de cognición a partir de los tres. Para obtener los resultados se calculó la proporción, considerando la cantidad de objetivos cumplidos y el total de los objetivos por edad.

Para los resultados cuantitativos obtenidos en la GPEP, se construyó una tabla de contingencia con los puntajes obtenidos por cada gemelo y se calculó $\chi^{2}$ con un nivel de confianza para $p<0.05$. El objetivo de ésta fue evaluar si existía o no diferencia significativa en el desempeño de las áreas evaluadas.

\section{Resultados}

Con respecto a los estudios realizados para evaluar la agudeza auditiva, en la audiometría, los gemelos no presentaron respuesta a estímulos por debajo de 100 $\mathrm{dB}$; en la timpanometría, obtuvieron curvas tipo A de acuerdo con la clasificación de Jerger bilateral (normales); en las EOAsT, presentaron adecuada estabilidad e inadecuada reproductibilidad global, y por frecuencia para ambos oídos. En PEATC, no tuvieron respuesta a 100 dB bilateral; en PEAEE, el GF presentó respuesta únicamente en oído derecho en la frecuencia 1,000 $\mathrm{Hz}$ a $95 \mathrm{~dB}$ y el GM en oído izquierdo en $500 \mathrm{~Hz}$ a $100 \mathrm{~dB}$, determinándose el diagnóstico de HNS profunda bilateral.

En los estudios de neuroimagen, el estudio de RM mostró en el corte sagital T1 un adelgazamiento de la región posterior del CC en ambos pacientes. En el corte axial T2 el GF tuvo adecuada mielinización de estructuras, mientras que en el GM fueron visibles zonas de mayor intensidad de señal parietoccipital bilateral, lo que indicó inadecuada mielinización y atrofia cortical frontoparietal bilateral; en la secuencia FIESTA (fast imaging employing steady state), a nivel del conducto auditivo interno, ambos mostraron adecuada emergencia de nervio coclear; el resto de estructuras se presentó sin alteración.

La evaluación por el Servicio de Oftalmología determinó diagnóstico de hipermetropía y miopía en ambos gemelos que fue corregido mediante lentes correctivos. En el caso del GM, se agregó estrabismo divergente de predominio derecho, el cual fue corregido quirúrgicamente.

Con respecto a la EDP-BL, el GF presentó control de postura y movilidad de acuerdo con la edad cronológica, así como discretas fallas en coordinación motriz fina, atención y concentración; el lenguaje lo establecía a nivel de balbuceo y su comunicación era apoyada en señas y gestos caseros, el cual se calificó muy por debajo de su edad con un nivel de desarrollo considerado inferior a lo normal. El GM presentó control de postura y movilidad por debajo de su edad, incoordinación y torpeza en motricidad gruesa y fina, comunicación limitada a llanto y balbuceo, aportando datos de ansiedad, timidez, inseguridad y dificultad para establecer relaciones interpersonales; asimismo, se reportó con retraso de desarrollo grave (Tabla 2).

En la GPEP, el GF demostró mayor destreza en las áreas del lenguaje, cognición y en el área motriz en comparación con el GM. Las ponderaciones más altas en el área del lenguaje fueron obtenidas para la edad de dos a tres años; en este caso, el GF obtuvo 14 de 30 objetivos (0.4666), mientras que el GM obtuvo siete de 30 (0.2333); en cognición, tuvieron un mejor desempeño durante la edad de tres a cuatro años: el GF con 15 de 23 objetivos (0.6521) y el GM con dos de 23 objetivos (0.0869); en el área motriz, para la edad de

Tabla 3: Resultados obtenidos en la Guía Portage de Educación Preescolar.

\begin{tabular}{lcccccc} 
& \multicolumn{2}{c}{$2-3$ años } & \multicolumn{2}{c}{$3-4$ años } & \multicolumn{2}{c}{$4-5$ años } \\
Área (objetivos) & GF & GM & GF & GM & GF & GM \\
\hline Lenguaje & $14 / 30=0.4666$ & $7 / 30=0.2333$ & $4 / 12=0.3333$ & $1 / 12=0.0833$ & $1 / 15=0.0666$ & $0 / 15=0$ \\
Cognición & - & - & $15 / 23=0.6521$ & $2 / 23=0.0869$ & $5 / 22=0.2272$ & $0 / 22=0$ \\
Desarrollo motriz & - & - & - & - & $10 / 16=0.625$ & $4 / 16=0.25$ \\
Socialización & - & - & - & - & $6 / 9=0.6666$ & $6 / 9=0.6666$ \\
\hline
\end{tabular}

$\mathrm{GF}$ = gemelo femenino; GM = gemelo masculino . 
cuatro a cinco años, el GF obtuvo 10 de 16 objetivos y el GM 4 de 16 objetivos (0.25). Finalmente, en el área de socialización ambos presentaron igual desempeño para la edad de cuatro a cinco años, obteniendo seis de nueve objetivos (0.6666) (Tabla 3).

Al realizar la tabla de contingencia y la prueba de $\chi^{2}$, se encontró diferencia significativa entre el GF y el GM en las evaluaciones de desarrollo cognitivo y del lenguaje con un nivel de significancia para $p<0.05$.

\section{DisCUSIÓN}

El interés por estudiar a estos pacientes surgió por la inquietud de conocer la evolución de su neurodesarrollo individual, tomando en cuenta la presencia de diversas entidades patológicas registradas en sus expedientes clínicos. Nos referimos a la HNS, al problema visual, a los hallazgos obtenidos en la RM, al grado de desarrollo reportado después de la valoración con la EDP-BL y la GPEP y, en cierta medida, al hecho de que fueran gemelos dicigóticos de diferente sexo.

Con respecto a la HNS, ambos pacientes presentaron deficiencia auditiva similar, la cual fue corregida en el oído derecho mediante IC con estricto seguimiento audiológico; en el caso de la problemática visual, ambos pacientes recibieron las ayudas correctivas necesarias para favorecer una adecuada función visual. Estos esfuerzos se encaminaron en resolver las importantes deficiencias en estas dos vías (visual y auditiva), ambas importantes y complementarias en la percepción del mundo y necesarias para el óptimo desarrollo cognitivo y del lenguaje. De alguna manera, al realizarlas, favorecieron la evolución del neurodesarrollo; sin embargo, es posible que se haya visto seriamente limitado por los hallazgos de los estudios de neuroimagen, en donde se evidenció un adelgazamiento de la región posterior en ambos gemelos, y en el caso del GM, deficiente mielinización y atrofia cortical frontoparietal bilateral.

Es evidente que el GM fue el más afectado, pues abarcó deficiencias más severas que inciden en aspectos no sólo estructurales, sino también funcionales, lo cual ha afectado de manera importante su neurodesarrollo. En el caso de la EDP-BL, aplicada a la edad de ingreso de los pacientes al INRLGII (1 año, 9 meses), se obtuvieron datos de retraso significativo del desarrollo en ambos gemelos en todas las áreas evaluadas, en especial en el GM. Los resultados arrojados por la GPEP aplicada a la edad de cuatro años seis meses muestran mejoría en el desempeño de ambos gemelos; sin embargo, éste sigue siendo más limitado y deficiente en el GM. Se observa que el GF obtiene un mejor desempeño en las áreas del lenguaje, cognición y motriz, aunque aún por debajo de lo esperado para su edad cronológica. Pese a ello, el GM muestra evolución deficiente al obtener puntajes ubicados muy por debajo a lo esperado para su edad cronológica; lo destacable es que ambos gemelos presentaron un grado de desarrollo normal en el área de socialización.

La edad en la que los pacientes recibieron el IC (dos años y 11 meses) es considerada "edad temprana", la cual, de acuerdo con lo reportado en la literatura, $6,8,9,12$ favorece el desarrollo del lenguaje $y$, por ende, el desarrollo cognitivo. Es posible que de no haber tenido acceso a esta ayuda auditiva a edad temprana, el desarrollo integral de ambos pacientes hubiera sido aún más pobre y limitado; aunado a esto, vale la pena retomar también lo reportado con respecto a las deficiencias oftalmológicas asociadas con discapacidad auditiva, pues la literatura estipula que esta doble discapacidad tiene serios efectos en el desarrollo de las habilidades de comunicación y en las habilidades cognitivas, ya que estos pacientes son extremadamente dependientes de la visión para compensar la pérdida auditiva. ${ }^{10,11}$ Por esta razón, es importante destacar la presencia de esta doble discapacidad en los gemelos; en ambos casos, las deficiencias fueron corregidas. A pesar de ello, el GM presentaba mayor desventaja y mayor complicación al agregarse el problema del estrabismo divergente con necesidad de corrección quirúrgica, ambas deficiencias, en especial el estrabismo, situación que sumó una dificultad, la cual evidentemente limitó en mayor medida el acceso a la información por esta vía.

Algo que nos parece fundamental destacar se relaciona con el medio ambiente, abarcando el social/ familiar y el educativo/terapéutico. Si consideramos el ambiente social/familiar, los gemelos conviven y se relacionan en el mismo núcleo, por lo que se espera que dentro del contexto en el que se desenvuelven sea prácticamente el mismo, es decir, han estado expuestos a los mismos estilos de crianza y de estimulación y en contacto con las mismas personas, así como con las mismas normas y principios. Respecto al ámbito educativo/terapéutico, ambos pacientes ingresaron a terapia del lenguaje bajo el mismo esquema de intervención (terapia auditivo verbal), atendidos por la misma terapista del lenguaje y asistieron durante el mismo periodo a sesiones terapéuticas, lo que de alguna manera nos permite sugerir que los ambientes en los que se desenvuelven los ubica en igualdad de condiciones. Esto también ha jugado un papel importante en la evolución de su neurodesarrollo; sin 
embargo, aunque es similar, debido a sus diferencias funcionales y anatómicas individuales no ha sido suficiente para lograr un desarrollo que se nivele con la edad cronológica.

Con respecto al sexo de los pacientes, considerando el dimorfismo sexual del SNC, podríamos suponer que la GF nuevamente se ve más favorecida (recordemos que en la literatura se estipula que en el encéfalo femenino se evidencian mayores procesos neurales en ambos hemisferios para las funciones del lenguaje caracterizadas por una mayor arborización dendrítica); también se observó una mayor densidad de las fibras nerviosas y una porción posterior del esplenio del CC más bulboso y significativamente mayor. ${ }^{14}$

\section{CONCLUSiones}

Ambos pacientes presentaban similares problemáticas funcionales y estructurales; sin embargo, el GF presentó menor severidad, lo que, de alguna manera, favoreció la evolución en su neurodesarrollo. Aunado a esto, vale la pena considerar las ventajas conocidas ya hace tiempo: que las niñas tienen mejor protección neuronal y son menos proclives a presentar patologías, o presentarlas con menor severidad, lo cual, en nuestro caso, la proveyó de mayor ventaja en comparación con su hermano.

Estudiar a poblaciones de gemelos es enriquecedor y trascendental, ya que nos aportan información valiosa al facilitar, de alguna manera, un mayor control de las variables, puesto que su situación suele ser la misma y también nos asegura una similaridad en cuestiones ambientales, educativas y atencionales.

\section{Bibliografía}

1. Hazan F, Ozturk AT, Adibelli H, Unal N, Tukun A. A novel missense mutation of the paired box 3 gene in a Turkish family with Waardenburg syndrome type 1. Mol Vis. 2013; 19: 196202.

2. Vichare N, Bhargava N. Waardenburg syndrome: A rare case with bilateral congenital cataract: An unusual entity. Med J Armed Forces India. 2013; 69: 172-174.

3. Kumar S, Rao K. Waardenburg syndrome: a rare genetic disorder, a report of two cases. Indian J of Hum Genet. 2012; 18: 254-255.
4. Akal A, Göncü T, Boyaci N, Yılmaz ÖF. Anisometropic amblyopia in a case of type 2 Waardenburg syndrome. BMJ Case Rep. 2013; 2013: bcr2013201140.

5. Madiha M, Khaled K. Waardenburg syndrome. Pan Afr Med J. 2015; 20: 427.

6. Koyama H, Kashio A, Sakata A et al. The hearing outcomes of cochlear implantation in Waardenburg syndrome. Biomed Res Int. 2016; 2016: 2854736.

7. de Sousa Andrade SM, Monteiro AR, Martins JH, Alves MC, Santos Silva LF, Quadros JM et al. Cochlear implant rehabilitation outcomes in Waardenburg syndrome children. Int J Pediatr Otorhinolaryngol. 2012; 76: 1375-1378.

8. Chen Y, Yang F, Zheng H, Zhou J, Zhu G, Hu P et al. Clinical and genetic investigation of families with type II Waardenburg syndrome. Mol Med Rep. 2016; 13: 1983-1988.

9. Magalhães AT, Samuel PA, Goffi-Gomez MV, Tsuji RK, Brito R, Bento RF. Audiological outcomes of cochlear implantation in Waardenburg syndrome. Int Arch Otorhinolaryngol. 2013; 17: 285-290.

10. Köylü MT, Gökçe G, Sobaci G, Oysul FG, Akincioğlu D. Ophthalmic pathologies in female subjects with bilateral congenital sensorineural hearing loss. Turk J Med Sci. 2016; 46: 139-144.

11. Falzon K, Guerin M, Fulcher T, Viani L. Ophthalmological screening of a paediatric cochlear implant population: a retrospective analysis and 12-year follow-up. Eye (Lond). 2010; 24: 1031-1036.

12. Ruggirello C, Mayer C. Language development in a hearing and a deaf twin with simultaneous bilateral cochlear implants. J Deaf Stud Deaf Educ. 2010; 15: 274-286.

13. Parra L, García AA, Ortiz S, Pérez D, Nájera J, Basurto NE et al. Las diferencias anatómicas cerebrales que implican diferencias funcionales (1a. de dos partes). Rev Fac Med UNAM. 2009; 52: 177-181.

14. Liu F, Vidarsson L, Winter JD, Tran H, Kassner A. Sex differences in the human corpus callosum microstructure: a combined T2 myelin-water and diffusion tensor magnetic resonance imaging study. Brain Res. 2010; 1343: 37-45.

15. Brunet O, Lezine I. El desarrollo psicológico de la primera infancia. Madrid: Pablo del Río; 1980.

16. Bluma S, Shearer M, Frohman A, Hilliard J. Guía Portage de educación preescolar. Wisconsin, EUA: Cooperative Educational Service Agency; 1978.

Correspondencia:

Ana Luisa Lino-González

Instituto Nacional de Rehabilitación

"Luis Guillermo Ibarra Ibarra".

Calzada México-Xochimilco Núm. 289,

Col. Arenal de Guadalupe, 14389,

Alcaldía de Tlalpan, Ciudad de México, México.

E-mail: ana_onil@yahoo.com.mx 